\title{
Adherence to preventive health care in children and young adults with Down syndrome (DS) in Sri Lanka
}

\author{
M P Senanayake, J A S S K Jayasinghe \\ (Index words: Down syndrome, Sri Lanka, complications)
}

\begin{abstract}
Objectives To assess adherence to preventive care and age-appropriate screening in different age categories of Down syndrome (DS) persons.

Methods This community-based retrospective observational study on ambulatory persons with DS from different regions of Sri Lanka was carried out on 100 randomly selected individuals from a sample framework of approximately 300 . Their screening behavior for cardiac, ophthalmic, hearing and thyroid status was evaluated. Age at first medical consultation for DS specific complications, whether undertaken when symptomatic or asymptomatic, and use of DS-specific growth charts were extracted from medical records. Screening was compared in three age groups $(<10,10-15$, and $>15$ years).

Results One hundred clinically diagnosed DS individuals (F: $M$ 1:1.2) aged 2-28 years (mean 13.5 years) were from varied socio-economic backgrounds and rural $(68 \%)$ suburban $(20 \%)$ and urban $(12 \%)$ settings. They all had had postnatal medical assessments. Initial eye, ENT, cardiac consultations was at significantly earlier ages in those below 10 years. Age-appropriate cardiac assessments occurred in $58 \%, 25 \%$ and $7.5 \%$ in each age group. Vision, hearing and newborn thyroid status were not routinely screened for. Only $7 \%$ had special growth charts. Area of residence had no effect on screening behavior.

Conclusions Screening for complications in DS was delayed and inconsistent but occurred at significantly earlier ages in younger children. Use of appropriate growth charts was very low. Screening behavior was unaffected by area of residence. We recommend incorporating DS-specific medical checklists as a preventive health routine for this unique group of children.
\end{abstract}

Ceylon Medical Journal 2014; 59: 13-15

\section{Introduction}

Survival of children with Down syndrome (DS) has improved around the world due to early detection of this syndrome's numerous multi-organ complications (1-4). Timely preventive health screening protocols are accepted health policy in many countries (5-6). Although preventive medical checklists with local adaptations, were advocated they are still not widely used in Sri Lanka (7). The objective of this study was to assess adherence to preventive health care in a sample of ambulatory DS persons and compare screening behaviour in different age categories.

\section{Methods}

This community-based retrospective observational study, was carried out on 100 ambulatory children and young persons (2-28 years) with DS, randomly selected from approximately 300 DS individuals attending a free health camp on Down Syndrome Day 2013 in Colombo. Due to logistical constraints, sample size was limited to 100 persons who provided consent from accompanying adult/s and had comprehensive medical records available for scrutiny. Absence of written medical records was an exclusion criterion.

An interviewer-administered questionnaire recorded age, gender, places of birth and residence, dates of first and subsequent medical consultations for cardiac, ophthalmic, hearing and thyroid assessments. Whether symptomatic or asymptomatic at first consultations, identified co-morbidities and availability of a special growth chart were obtained from medical records. Patient demographics and screening behaviour were compared between the three age groups $(2-<10$ years, $10-15$ years and $>15$ years) using $Z$ test for two sample means/ proportions.

\section{Results}

There were 100 clinically diagnosed DS individuals (44 females 56 males, F: M ratio 1:1.2) aged 2-28 years (mean 13.5 years) of varied socio-economic backgrounds. Area of residence were rural (68) suburban (20) urban (12). Entire sample was hospital-born and had postnatal medical examinations.

Department of Paediatrics, Faculty of Medicine, University of Colombo, Sri Lanka.

Correspondence: MPS, e-mail: <manouri.senanayake@gmail.com>. Received 8 July 2013 and revised version accepted 27 September 2013. Competing interests: none declared. 
Timing of investigations for DS specific complications and identified co-morbidities are given in Table 1. Cardiac assessment including of echocardiography was done in $66 \%$. Age at first echocardiogram ranged from neonate to 17 years. Cardiac assessment was significantly earlier in younger age groups $(p<0.05)$ (Table 2 ) but only $29 \%$ had echocardiography by the recommended age of three months. Those $<10$ years had highest coverage $(58 \%)$ but was a preventive measure only in a minority (32\%). Others were assessed because of "heart murmur" (61\%), "breathlessness" (5\%), and "recurrent chest infections" (3\%). Heart defects were documented in 43 persons.

Ophthalmic assessment was done in $60 \%$ of whom $58 \%$ were asymptomatic. Squint, nystagmus and nasolacrimal duct obstruction were the reasons for seeking ophthalmic services. First assessment was carried out by six months of age in only $7 \%$ and statistically significant $(p<0.05)$ were seen between age groups (Table 2$)$. In 35\% ophthalmic assessment was after age 10 years. Only 48\% had ENT assessments and of whom 69\% had hearing assessed. Severe deafness was reported in 9\%. Main reasons for ENT consultations were infections. Only $8 \%$ were assessed in infancy. Ophthalmic or audiological assessments did not take place annually. Thirty seven underwent thyroid hormone assay and 13 (35\%) were on treatment for hypothyroidism.

Screening behavior in the three age groups found significant differences $(p<0.05)$ in age of initial cardiac, eye and hearing assessments; and screening rates were highest in the youngest age group (Table 2). Area of residence showed no relationship $(p>0.05)$ to screening behavior. Special DS-growth charts were used in $7 \%$.

\section{Table 1. Screening behaviour and co-morbidities in 100 Down Syndrome individuals aged 2-28 years}

\begin{tabular}{ll}
\hline Screening behaviour & Number $(\%)$ \\
\hline Echocardiography performed at least once $(\mathrm{n}=100)$ & $66(66 \%)$ \\
Echocardiography by age 3 months $(\mathrm{n}=100)$ & $29(29 \%)$ \\
Mean age of first echocardiogram $(\mathrm{n}=66)$ & 3.2 years \\
Reason for echocardiography $(\mathrm{n}=66)$ & \\
Routine screening when asymptomatic & $21(31.8 \%)$ \\
Cardiac murmur & $40(60.6 \%)$ \\
Breathlessness & $3(4.5 \%)$ \\
Recurrent chest infections & $2(3.0 \%)$ \\
Cyanosis & $0(0 \%)$ \\
Heart defects identified $(\mathrm{n}=66)$ & $43(65 \%)$
\end{tabular}

Type of heart lesion/s $(n=43)$

$\begin{array}{ll}\text { ASD } & 16(37 \%) \\ \text { VSD } & 13(30 \%) \\ \text { PDA } & 8(19 \%) \\ \text { AV canal defects } & 3(7 \%) \\ \text { Complex cardiac lesions } & 12(28 \%) \\ \text { Heart surgery needed } & 22(51.2 \%)\end{array}$

At least one eye assessment performed $(n=100)$

$60(60 \%)$

Mean age of initial eye assessment $(n=60)$

9.0 years

$(\mathrm{SD}=7.5$

years)

Eye assessment by age 6 months $(n=60)$

$4(7 \%)$

Reason for eye assessment $(n=60)$

$$
\begin{aligned}
& \text { screening when asymptomatic } \\
& \text { squint, nystagmus, blocked }
\end{aligned}
$$

naso-lacrimal duct etc

Reason for eye check by six months infancy $(n=4)$

$$
\text { abnormality detected by paediatrician } \quad 4(100 \%)
$$

Age at initial eye assessment

$$
\begin{array}{cl}
\text { Infancy } & 4(6.7 \%) \\
1-5 \text { years } & 20(33.3 \%) \\
5-10 \text { years } & 15(25 \%) \\
>10 \text { years } & 21(35 \%) \\
\text { Number identified with eye abnormalities }(\mathrm{n}=60) & 37(61.7 \%) \\
\text { Needed spectacles } & 27(45 \%) \\
\text { Needed surgery } & 2(3.3 \%)
\end{array}
$$

Annual check of vision $\quad 0$

Number who sought ENT services $(n=100) \quad 48(48 \%)$

ENT assessment by six months $(n=100) \quad 4(4 \%)$

Number who had at least one hearing

assessment $(\mathrm{n}=48)$

$33(68.8 \%)$

Severe deafness

$3(9.1 \%)$

Age at initial ENT assessment $(n=48)$

Infancy
$1-5$ years
$5-10$ years
$>10$ years

$4(8.3 \%)$

$22(45.8 \%)$

$11(22.9 \%)$

$11(22.9 \%)$

Mean age of hearing assessment $(n=33) \quad 6.7$ years

$(\mathrm{SD}=6.2$

years)

Audiology annually 0

Thyroid status assessed $(n=100) \quad 37(37 \%)$

Neonatal screening $(\mathrm{n}=37)$

Hypothyroidism identified $(\mathrm{n}=37)$

$13(35.1 \%)$ 
Table 2. Comparison of screening / medical checks in three age groups in Down syndrome

\begin{tabular}{|c|c|c|c|}
\hline & $=/<10$ years $(n=33)$ & $>10-15$ years $(n=28)$ & $>15$ years $(n=38)$ \\
\hline Mean age of initial echocardiogram & 8.2 months & 35.8 months & 76.6 months \\
\hline Echocardiography by 3 months of age & $19(57.6 \%)$ & $7(25 \%)$ & $3(7.9 \%)$ \\
\hline Mean age at initial eye assessment & $3.2 \mathrm{Y}$ & $6.67 \mathrm{Y}$ & $14.96 \mathrm{Y}$ \\
\hline Initial eye check at/before 6 months & $3(9.1 \%)$ & 0 & $1(2.63 \%)$ \\
\hline Mean age of initial ENT encounter & $2.33 \mathrm{Y}$ & $5.54 \mathrm{Y}$ & $12.53 \mathrm{Y}$ \\
\hline Initial ENT consultation by 6 months & $4(12.1 \%)$ & 0 & 0 \\
\hline Neonatal thyroid screen & 0 & 0 & 0 \\
\hline TH assay during infancy & $16(48.5 \%)$ & $13(46.4 \%)$ & $7(18.4 \%)$ \\
\hline
\end{tabular}

\section{Discussion}

In the absence of a national registry for DS in Sri Lanka, we used a registry of 350 DS persons, maintained by a national level non-governmental organization. Our sample framework consisted of approximately 300 DS persons of this registry who attended a health camp. Lack of medical records was not a significant problem in this sample. Preventive health checks on four organs that commonly affect quality of life in DS (heart, eye, ear and thyroid) were retrospectively investigated for, in this sample of 100 randomly selected DS individuals.

We found low screening rates and a substantial proportion without cardiac $(34 \%)$, ophthalmic $(40 \%)$ or hearing (67\%) assessments. Annual ophthalmic and audiology screening which is invaluable during school years had not taken place at all. Even in the youngest age group which had the best cardiac screening rates, initial assessment was delayed in $42 \%$. DS-specific growth charts were used only in a small minority. Access to medical facilities did not appear to be a contributing factor because there was no difference between rural and urban children.

Screening rates in DS persons not included in a registry may be even lower than those found in this study. We recommend a special preventive health program for DS children, incorporating DS-specific screening checklists and growth charts, to improve their health status and quality of life.

\section{Acknowledgements}

We thank Kosala Dullewa Foundation for permission to conduct this survey and Rotary Club Colombo District, Interact Club of Royal College Colombo and the team of volunteer doctors for their invaluable assistance.

\section{References}

1. Kucik JE, Shin M, Siffel C, Marengo L, Correa A. Trends in survival among children with Down syndrome in 10 regions of the United States. Pediatrics 2013; 131: 27-36.

2. Zhu JL, Hasle H, Correa A, et al. Survival among people with Down syndrome: a nationwide population-based study in Denmark. Genetics in Medicine 2013; 15: 64-9.

3. Jensen KM, Taylor LC, Davis MM. Primary care for adults with Down syndrome: adherence to preventive healthcare recommendations. Journal of Intellectual Disability Research 2013; 57: 409-21.

4. Wilson L. Preventive Care for Adults With Down Syndrome. American College of Preventive Medicine 2010. Available at http://www.medscape.org/viewarticle/715382.

5. European Down Syndrome Association, Health Care Guidelines for People With Down Syndrome, Available at http://www.edsa.eu/files/essentials/edsa_ essentials_ 2 healthcare.pdf

6. American Academy of Pediatrics Committee on Genetics. Health supervision for children with Down syndrome. Pediatrics 2001; 107: 442-50.

7. Senanayake MP. Caring for children with Down syndrome: a medical checklist. Sri Lanka Journal of Child Health 2008; 37: 17-9. 